\title{
Differential equations driven by Hölder continuous functions of order greater than $1 / 2$
}

\author{
Yaozhong $\mathrm{Hu}^{*}$ and David Nualart \\ Department of Mathematics, University of Kansas \\ 405 Snow Hall, Lawrence, Kansas 66045-2142
}

\begin{abstract}
We derive estimates for the solutions to differential equations driven by a Hölder continuous function of order $\beta>1 / 2$. As an application we deduce the existence of moments for the solutions to stochastic partial differential equations driven by a fractional Brownian motion with Hurst parameter $H>\frac{1}{2}$.
\end{abstract}

\section{Introduction}

We are interested in the solutions of differential equations on $\mathbb{R}^{m}$ of the form

$$
x_{t}=x_{0}+\int_{0}^{t} f\left(x_{r}\right) d y_{r}
$$

where the driving force $y:[0, \infty) \rightarrow \mathbb{R}^{m}$ is a Hölder continuous function of order $\beta>1 / 2$. If the function $f: \mathbb{R}^{d} \rightarrow \mathbb{R}^{m d}$ has bounded partial derivatives which are Hölder continuous of order $\lambda>\frac{1}{\beta}-1$, then there is a unique solution $x: \mathbb{R}^{m} \rightarrow \mathbb{R}$, which has bounded $\frac{1}{\beta}$-variation on any finite interval. These results have been proved by Lyons in 2] using the $p$-variation norm and the technique introduced by Young in [6. The integral appearing in (1.1) is then a Riemann-Stieltjes integral.

In [7] Zähle has introduced a generalized Stieltjes integral using the techniques of fractional calculus. This integral is expressed in terms of fractional derivative operators and it coincides with the Riemann-Stieltjes integral $\int_{0}^{T} f d g$, when the functions $f$ and $g$ are Hölder continuous of orders $\lambda$ and $\mu$, respectively and $\lambda+\mu>1$ (see Proposition 2.1 below). Using this formula for the RiemannStieltjes integral, Nualart and Răşcanu have obtained in 3 the existence of a unique solution for a class of general differential equations that includes (1.1). Also they have proved that the solution of (1.1) is bounded on a finite interval

${ }^{*}$ Y. $\mathrm{Hu}$ is supported in part by the National Science Foundation under Grant No. DMS0204613 and DMS0504783 
$[0, T]$ by $C_{1} \exp \left(C_{2}\|y\|_{0, T, \beta}^{\kappa}\right)$, where $\kappa>\frac{1}{\beta}$ if $f$ is bounded and $\kappa>\frac{1}{1-2 \beta}$ is $f$ has linear growth. Here $\|y\|_{0, T, \beta}$ denotes the $\beta$-Hölder norm of $y$ on the time interval $[0, T]$. These estimates are based on a suitable application of Gronwall's lemma. It turns out that the estimate in the linear growth case is unsatisfactory because $\kappa$ tends to infinity as $\beta$ tends to $1 / 2$.

The main purpose of this paper is to obtain better estimates for the solution $x_{t}$ in the case where $f$ is bounded or has linear growth using a direct approach based on formula (2.8). In the case where $f$ is bounded we estimate $\sup _{0 \leq t \leq T}\left|x_{t}\right|$ by

$$
C\left(1+\|y\|_{0, T, \beta}^{\frac{1}{\beta}}\right)
$$

and if $f$ has linear growth we obtain the estimate

$$
C_{1} \exp \left(C_{2}\|y\|_{0, T, \beta}^{\frac{1}{\beta}}\right) .
$$

In Theorem 3.1 we provide explicit dependence on $f$ and $T$ for the constants $C$, $C_{1}$ and $C_{2}$.

Another novelty of this paper is that we establish the explicit dependence of the solution $x_{t}$ to (1.1) on the initial condition $x_{0}$, the driving control $y$ and the coefficient $f$ (Theorem 3.2). Similar results are obtained for the case $1 / 3<\beta<1 / 2$ in a forthcoming paper [1].

As an application we deduce the existence of moments for the solutions to stochastic partial differential equations driven by a fractional Brownian motion with Hurst parameter $H>\frac{1}{2}$. We also discuss the regularity of the solution in the sense of Malliavin Calculus, improving the results of Nualart and Saussereau 4, and we apply the techniques of the Malliavin calculus to establish the existence of densities under suitable non-degeneracy conditions.

\section{Fractional integrals and derivatives}

Let $a, b \in \mathbb{R}$ with $a<b$. Let $f \in L^{1}(a, b)$ and $\alpha>0$. The left-sided and right-sided fractional Riemann-Liouville integrals of $f$ of order $\alpha$ are defined for almost all $x \in(a, b)$ by

$$
I_{a+}^{\alpha} f(t)=\frac{1}{\Gamma(\alpha)} \int_{a}^{t}(t-s)^{\alpha-1} f(s) d s
$$

and

$$
I_{b-}^{\alpha} f(t)=\frac{(-1)^{-\alpha}}{\Gamma(\alpha)} \int_{t}^{b}(s-t)^{\alpha-1} f(s) d s,
$$

respectively, where $(-1)^{-\alpha}=e^{-i \pi \alpha}$ and $\Gamma(\alpha)=\int_{0}^{\infty} r^{\alpha-1} e^{-r} d r$ is the Euler gamma function. Let $I_{a+}^{\alpha}\left(L^{p}\right)$ (resp. $I_{b-}^{\alpha}\left(L^{p}\right)$ ) be the image of $L^{p}(a, b)$ by the 
operator $I_{a+}^{\alpha}$ (resp. $\left.I_{b-}^{\alpha}\right)$. If $f \in I_{a+}^{\alpha}\left(L^{p}\right) \quad\left(\right.$ resp. $\left.f \in I_{b-}^{\alpha}\left(L^{p}\right)\right)$ and $0<\alpha<1$ then the Weyl derivatives are defined as

$$
D_{a+}^{\alpha} f(t)=\frac{1}{\Gamma(1-\alpha)}\left(\frac{f(t)}{(t-a)^{\alpha}}+\alpha \int_{a}^{t} \frac{f(t)-f(s)}{(t-s)^{\alpha+1}} d s\right)
$$

and

$$
D_{b-}^{\alpha} f(t)=\frac{(-1)^{\alpha}}{\Gamma(1-\alpha)}\left(\frac{f(t)}{(b-t)^{\alpha}}+\alpha \int_{t}^{b} \frac{f(t)-f(s)}{(s-t)^{\alpha+1}} d s\right)
$$

where $a \leq t \leq b$ (the convergence of the integrals at the singularity $s=t$ holds point-wise for almost all $t \in(a, b)$ if $p=1$ and moreover in $L^{p}$-sense if $1<p<\infty)$.

For any $\lambda \in(0,1)$, we denote by $C^{\lambda}(a, b)$ the space of $\lambda$-Hölder continuous functions on the interval $[a, b]$. We will make use of the notation

$$
\|x\|_{a, b, \beta}=\sup _{a \leq \theta<r \leq b} \frac{\left|x_{r}-x_{\theta}\right|}{|r-\theta|^{\beta}},
$$

and

$$
\|x\|_{a, b, \infty}=\sup _{a \leq r \leq b}\left|x_{r}\right|,
$$

where $x: \mathbb{R}^{d} \rightarrow \mathbb{R}$ is a given continuous function.

Recall from [5] that we have:

- If $\alpha<\frac{1}{p}$ and $q=\frac{p}{1-\alpha p}$ then

$$
I_{a+}^{\alpha}\left(L^{p}\right)=I_{b-}^{\alpha}\left(L^{p}\right) \subset L^{q}(a, b) .
$$

- If $\alpha>\frac{1}{p}$ then

$$
I_{a+}^{\alpha}\left(L^{p}\right) \cup I_{b-}^{\alpha}\left(L^{p}\right) \subset C^{\alpha-\frac{1}{p}}(a, b) .
$$

The following inversion formulas hold:

$$
\begin{aligned}
I_{a+}^{\alpha}\left(D_{a+}^{\alpha} f\right)=f, & \forall f \in I_{a+}^{\alpha}\left(L^{p}\right) \\
I_{a-}^{\alpha}\left(D_{a-}^{\alpha} f\right)=f, & \forall f \in I_{a-}^{\alpha}\left(L^{p}\right)
\end{aligned}
$$

and

$$
D_{a+}^{\alpha}\left(I_{a+}^{\alpha} f\right)=f, \quad D_{a-}^{\alpha}\left(I_{a-}^{\alpha} f\right)=f, \quad \forall f \in L^{1}(a, b) .
$$

On the other hand, for any $f, g \in L^{1}(a, b)$ we have

$$
\int_{a}^{b} I_{a+}^{\alpha} f(t) g(t) d t=(-1)^{\alpha} \int_{a}^{b} f(t) I_{b-}^{\alpha} g(t) d t,
$$

and for $f \in I_{a+}^{\alpha}\left(L^{p}\right)$ and $g \in I_{a-}^{\alpha}\left(L^{p}\right)$ we have

$$
\int_{a}^{b} D_{a+}^{\alpha} f(t) g(t) d t=(-1)^{-\alpha} \int_{a}^{b} f(t) D_{b-}^{\alpha} g(t) d t .
$$


Suppose that $f \in C^{\lambda}(a, b)$ and $g \in C^{\mu}(a, b)$ with $\lambda+\mu>1$. Then, from the classical paper by Young [6], the Riemann-Stieltjes integral $\int_{a}^{b} f d g$ exists. The following proposition can be regarded as a fractional integration by parts formula, and provides an explicit expression for the integral $\int_{a}^{b} f d g$ in terms of fractional derivatives (see [7]).

Proposition 2.1 Suppose that $f \in C^{\lambda}(a, b)$ and $g \in C^{\mu}(a, b)$ with $\lambda+\mu>1$. Let $\lambda>\alpha$ and $\mu>1-\alpha$. Then the Riemann Stieltjes integral $\int_{a}^{b} f d g$ exists and it can be expressed as

$$
\int_{a}^{b} f d g=(-1)^{\alpha} \int_{a}^{b} D_{a+}^{\alpha} f(t) D_{b-}^{1-\alpha} g_{b-}(t) d t
$$

where $g_{b-}(t)=g(t)-g(b)$.

\section{Estimates for the solutions of differential equa- tions}

Suppose that $y:[0, \infty) \rightarrow \mathbb{R}^{m}$ is a Hölder continuous function of order $\beta>1 / 2$. Fix an initial condition $x_{0} \in \mathbb{R}^{d}$ and consider the following differential equation

$$
x_{t}=x_{0}+\int_{0}^{t} f\left(x_{r}\right) d y_{r},
$$

where $f: \mathbb{R}^{d} \rightarrow \mathbb{R}^{m d}$ is given function. Lyons has proved in 2] that Equation (3.1) has a unique solution if $f$ is continuously differentiable and it has a derivative $f^{\prime}$ which is bounded and locally Hölder continuous of order $\lambda>\frac{1}{\beta}-1$.

Our aim is to obtain estimates on $x_{t}$ which are better than those given by Nualart and Răşcanu in 3 .

Theorem 3.1 Let $f$ be a continuously differentiable such that $f^{\prime}$ is bounded and locally Hölder continuous of order $\lambda>\frac{1}{\beta}-1$.

(i) Assume that $f$ is also bounded. Then, there is a constant $k$, which depends only on $\beta$, such that for all $T$,

$$
\sup _{0 \leq t \leq T}\left|x_{t}\right| \leq\left|x_{0}\right|+k T\|f\|_{\infty}\left\|f^{\prime}\right\|_{\infty}^{\frac{1-\beta}{\beta}}\|y\|_{0, T, \beta}^{\frac{1}{\beta}}
$$

(ii) Assume that $f$ satisfies the linear growth condition

$$
|f(x)| \leq a_{0}+a_{1}|x|
$$

where $a_{0} \geq 0$ and $a_{1} \geq 0$. Then there is a constant $k$ depending only on $\beta$, such that for all $T$,

$$
\sup _{0 \leq t \leq T}\left|x_{t}\right| \leq 2^{k T\left[\left\|f^{\prime}\right\|_{\infty} \vee a_{0} \vee a_{1}\right]^{1 / \beta}\|y\|_{0, T, \beta}^{1 / \beta}\left(\left|x_{0}\right|+1\right) .}
$$


Proof. Without loss of generality we assume that $d=m=1$. Assume first that $f$ is bounded. Set $\|y\|_{\beta}=\|y\|_{0, T, \beta}$. Let $\alpha>1 / 2$ such that $\alpha>1-\beta$. First we use the fractional integration by parts formula given in Proposition 2.1 to obtain for all $s, t \in[0, T]$,

$$
\left|\int_{s}^{t} f\left(x_{r}\right) d y_{r}\right| \leq \int_{s}^{t}\left|D_{s+}^{\alpha} f\left(x_{r}\right) D_{t-}^{1-\alpha} y_{t-}(r)\right| d r .
$$

From (2.2) and (2.1) it is easy to see

$$
\left|D_{t-}^{1-\alpha} y_{t-}(r)\right| \leq k\|y\|_{r, t, \beta}|t-r|^{\alpha+\beta-1} \leq k\|y\|_{\beta}|t-r|^{\alpha+\beta-1}
$$

and

$$
\left|D_{s+}^{\alpha} f\left(x_{r}\right)\right| \leq k\left[\|f\|_{\infty}(r-s)^{-\alpha}+\left\|f^{\prime}\right\|_{\infty}\|x\|_{s, t, \beta}(r-s)^{\beta-\alpha}\right] .
$$

Therefore

$$
\begin{aligned}
\left|\int_{s}^{t} f\left(x_{r}\right) d y_{r}\right| \leq & k\|y\|_{\beta} \int_{s}^{t}\left[\|f\|_{\infty}(r-s)^{-\alpha}(t-r)^{\alpha+\beta-1}\right. \\
& \left.+\left\|f^{\prime}\right\|_{\infty}\|x\|_{s, t, \beta}(r-s)^{\beta-\alpha}(t-r)^{\alpha+\beta-1}\right] d r \\
\leq & k\|y\|_{\beta}\left[\|f\|_{\infty}(t-s)^{\beta}+\left\|f^{\prime}\right\|_{\infty}\|x\|_{s, t, \beta}(t-s)^{2 \beta}\right] .
\end{aligned}
$$

Consequently, we have

$$
\|x\|_{s, t, \beta} \leq k\|y\|_{\beta}\left[\|f\|_{\infty}+\left\|f^{\prime}\right\|_{\infty}\|x\|_{s, t, \beta}(t-s)^{\beta}\right] .
$$

Hence,

$$
\|x\|_{s, t, \beta} \leq k\|y\|_{\beta}\|f\|_{\infty}\left(1-k\left\|f^{\prime}\right\|_{\infty}\|y\|_{\beta}(t-s)^{\beta}\right)^{-1} .
$$

Therefore,

$$
\begin{aligned}
\|x\|_{s, t, \infty} & \leq\left|x_{s}\right|+\|x\|_{s, t, \beta}(t-s)^{\beta} \\
& \leq\left|x_{s}\right|+k\|y\|_{\beta}\|f\|_{\infty}\left(1-k\left\|f^{\prime}\right\|_{\infty}\|y\|_{\beta}(t-s)^{\beta}\right)^{-1}(t-s)^{\beta} .
\end{aligned}
$$

Let $A:=k\left\|f^{\prime}\right\|_{\infty}\|y\|_{\beta}$. Divide the interval $[0, T]$ into $n=T / \Delta$ subintervals and apply the above inequality on the interval $[0, \Delta],[\Delta, 2 \Delta]$ and so on recursively to obtain

$$
\sup _{0 \leq t \leq T}\left|x_{t}\right| \leq\left|x_{0}\right|+k T\|f\|_{\infty}\|y\|_{\beta}\left(1-A \Delta^{\beta}\right)^{-1} \Delta^{\beta-1} .
$$

With the choice $\Delta=\left(\frac{1-\beta}{A}\right)^{\frac{1}{\beta}}$ we get

$$
\begin{aligned}
\sup _{0 \leq t \leq T}\left|x_{t}\right| & \leq\left|x_{0}\right|+k T\|f\|_{\infty}\|y\|_{\beta} \frac{1}{\beta(1-\beta)^{\frac{1-\beta}{\beta}}}\left(k\left\|f^{\prime}\right\|_{\infty}\|y\|_{\beta}\right)^{\frac{1-\beta}{\beta}} \\
& =\left|x_{0}\right|+k T\left\|f^{\prime}\right\|_{\infty}^{\frac{1-\beta}{\beta}}\|y\|_{\beta}^{\frac{1}{\beta}} .
\end{aligned}
$$


This proves the inequality (3.2).

Assume now that $f$ satisfies (3.3). In this case, instead of (3.6) we have

$$
\left|D_{s+}^{\alpha} f\left(x_{r}\right)\right| \leq k\left[\left(a_{0}+a_{1}\left|x_{r}\right|\right)(r-s)^{-\alpha}+\left\|f^{\prime}\right\|_{\infty}\|x\|_{s, t, \beta}(r-s)^{\beta-\alpha}\right] .
$$

As a consequence,

$$
\|x\|_{s, t, \beta} \leq k\|y\|_{\beta}\left[a_{0}+a_{1}\|x\|_{s, t, \infty}+\left\|f^{\prime}\right\|_{\infty}\|x\|_{s, t, \beta}(t-s)^{\beta}\right] .
$$

Or

$$
\|x\|_{s, t, \beta} \leq k\|y\|_{\beta}\left(a_{0}+a_{1}\|x\|_{s, t, \infty}\right)\left(1-k\left\|f^{\prime}\right\|_{\infty}\|y\|_{\beta}(t-s)^{\beta}\right)^{-1} .
$$

Therefore,

$$
\begin{aligned}
\left|x_{t}\right| \leq & \left|x_{s}\right|+k\|y\|_{\beta}\left(1-k\left\|f^{\prime}\right\|_{\infty}\|y\|_{\beta}(t-s)^{\beta}\right)^{-1} \\
& \times\left(a_{0}+a_{1}\|x\|_{s, t, \infty}\right)(t-s)^{\beta}
\end{aligned}
$$

As before, divide the interval $[0, T]$ into $n=T / \Delta$ subintervals and set $\Delta=t-s$.

Denote

$$
\begin{aligned}
A & =k\left\|f^{\prime}\right\|_{\infty}\|y\|_{\beta} \\
B & =k a_{0}\|y\|_{\beta} \\
C & =k a_{1}\|y\|_{\beta} \\
D & =\left(1-\left(1-A \Delta^{\beta}\right)^{-1} C \Delta^{\beta}\right)^{-1} \\
F & =D B\left(1-A \Delta^{\beta}\right)^{-1} \Delta^{\beta} .
\end{aligned}
$$

We have

$$
\begin{aligned}
& \|x\|_{s, t, \infty}\left[1-k\|y\|_{\beta}\left(1-A \Delta^{\beta}\right)^{-1} a_{1} \Delta^{\beta}\right] \\
\leq & \left|x_{s}\right|+k a_{0}\|y\|_{\beta}\left(1-A \Delta^{\beta}\right)^{-1} \Delta^{\beta} .
\end{aligned}
$$

This implies

$$
\sup _{0 \leq r \leq t}\left|x_{r}\right| \leq\left(1-\left(1-A \Delta^{\beta}\right)^{-1} C \Delta^{\beta}\right)^{-1}\left[\sup _{0 \leq r \leq s}\left|x_{r}\right|+B\left(1-A \Delta^{\beta}\right)^{-1} \Delta^{\beta}\right] .
$$

Or

$$
\sup _{0 \leq r \leq t}\left|x_{r}\right| \leq D \sup _{0 \leq r \leq s}\left|x_{r}\right|+F
$$

Denote

$$
Z_{n}=\sup _{0 \leq r \leq n \Delta}\left|x_{r}\right|
$$

where $n=\frac{T}{\Delta}$. Then

$$
Z_{n} \leq D Z_{n-1}+F \leq \cdots \leq D^{n} Z_{0}+\sum_{k=0}^{n-1} D^{k} F
$$


This yields

$$
\begin{aligned}
\sup _{0 \leq t \leq T}\left|x_{t}\right| \leq & \left(1-\left(1-A \Delta^{\beta}\right)^{-1} C \Delta^{\beta}\right)^{-T / \Delta}\left|x_{0}\right| \\
& +\sum_{k=0}^{n-1}\left(1-\left(1-A \Delta^{\beta}\right)^{-1} C \Delta^{\beta}\right)^{-k-1} B\left(1-A \Delta^{\beta}\right)^{-1} \Delta^{\beta} .
\end{aligned}
$$

Then we let $\Delta$ satisfy

$$
A \Delta^{\beta} \leq 1 / 3, C \Delta^{\beta} \leq 1 / 3, B \Delta^{\beta} \leq 1 / 3
$$

Namely, we take

$$
\Delta=\left(\frac{1}{3(A \vee B \vee C)}\right)^{1 / \beta}
$$

Then

$$
\begin{aligned}
\sup _{0 \leq t \leq T}\left|x_{t}\right| & \leq 2^{T / \Delta}\left(\left|x_{0}\right|+1\right) \\
& \leq 2^{k T\left[\left\|f^{\prime}\right\|_{\infty} \vee a_{0} \vee a_{1}\right]^{1 / \beta}\|y\|_{0, T, \beta}^{1 / \beta}}\left(\left|x_{0}\right|+1\right) .
\end{aligned}
$$

The proof of the theorem is now complete.

Suppose now that we have two differential equations of the form

$$
x_{t}=x_{0}+\int_{0}^{t} f\left(x_{s}\right) d y_{s}
$$

and

$$
\tilde{x}_{t}=\tilde{x}_{0}+\int_{0}^{t} \tilde{f}\left(\tilde{x}_{s}\right) \tilde{y}_{s},
$$

where $y$ and $\widetilde{y}$ are Hölder continuous functions of order $\beta>1 / 2$, and $f$ and $\tilde{f}$ are two functions which are continuously differentiable with Hölder continuous derivatives of order $\lambda>\frac{1}{\beta}-1$. Then, we have the following estimate.

Theorem 3.2 Suppose in addition that $f$ is twice continuously differentiable and $f^{\prime \prime}$ is bounded. Then there is a constant $k$ such that

$$
\begin{aligned}
\sup _{0 \leq r \leq T}\left|x_{r}-\tilde{x}_{r}\right| \leq & 2^{k D^{1 / \beta}\|y\|_{0, T, \beta}^{1 / \beta} T} \\
& \times\left\{\left|x_{0}-\tilde{x}_{0}\right|+\|y\|_{0, T, \beta}\left[\|f-\tilde{f}\|_{\infty}+\|x\|_{0, T, \beta}\left\|f^{\prime}-\tilde{f}^{\prime}\right\|_{\infty}\right]\right. \\
& \left.+\left[\|f\|_{\infty}+\|\tilde{f}\|_{\infty}\|x\|_{0, T, \infty}\right]\|y-\tilde{y}\|_{0, T, \beta}\right\}
\end{aligned}
$$

where

$$
D=\left\|f^{\prime}\right\|_{\infty} \vee\left(\left\|f^{\prime}\right\|_{\infty}\|y\|_{0, T, \beta}+\left\|f^{\prime \prime}\right\|_{\infty}\left(\|x\|_{0, T, \beta}+\|\tilde{x}\|_{0, T, \beta}\right) T^{\beta}\right) .
$$


Proof. Fix $s, t \in[0, T]$. Set

$$
x_{t}-\tilde{x}_{t}-\left(x_{s}-\tilde{x}_{s}\right)=I_{1}+I_{2}+I_{3}
$$

where

$$
\begin{aligned}
I_{1} & =\int_{s}^{t}\left[f\left(x_{r}\right)-f\left(\tilde{x}_{r}\right)\right] d y_{r} \\
I_{2} & =\int_{s}^{t}\left[f\left(\tilde{x}_{r}\right)-\tilde{f}\left(\tilde{x}_{r}\right)\right] d y_{r} \\
I_{3} & =\int_{s}^{t} \tilde{f}\left(\tilde{x}_{r}\right) d\left[y_{r}-\tilde{y}_{r}\right] .
\end{aligned}
$$

The terms $I_{2}$ and $I_{3}$ can be estimated easily.

$$
\left|I_{2}\right| \leq k\|y\|_{\beta}\left[\|f-\tilde{f}\|_{\infty}(t-s)^{\beta}+\left\|f^{\prime}-\tilde{f}^{\prime}\right\|_{\infty}\|\tilde{x}\|_{s, t, \beta}(t-s)^{2 \beta}\right]
$$

and

$$
\left|I_{3}\right| \leq k\|y-\tilde{y}\|_{\beta}\left[\|\tilde{f}\|_{\infty}(t-s)^{\beta}+\left\|\tilde{f}^{\prime}\right\|_{\infty}\|\tilde{x}\|_{s, t, \beta}(t-s)^{2 \beta}\right],
$$

where $\|y\|_{\beta}=\|y\|_{0, T, \beta}$ and $\|y-\tilde{y}\|_{\beta}=\|y-\tilde{y}\|_{0, T, \beta}$. The term $I_{1}$ is a little more complicated.

$$
\begin{aligned}
\left|I_{1}\right| \leq & \int_{s}^{t}\left|D_{s+}^{\alpha}\left[f\left(x_{r}\right)-f\left(\tilde{x}_{r}\right)\right] \| D_{t-}^{1-\alpha} y_{t-}(r)\right| d r \\
\leq & k \int_{s}^{t}\|y\|_{s, t, \beta}(t-r)^{\alpha+\beta-1}\left[\left|f\left(x_{r}\right)-f\left(\tilde{x}_{r}\right)\right|(r-s)^{-\alpha}\right. \\
& \quad+\left\|f^{\prime}\right\|_{\infty}\|x-\tilde{x}\|_{s, r, \beta}(r-s)^{\beta-\alpha} \\
\quad & \left.\quad\left\|f^{\prime \prime}\right\|_{\infty}\|x-\tilde{x}\|_{s, r, \infty}\left[\|x\|_{s, r, \beta}+\|\tilde{x}\|_{s, r, \beta}\right](r-s)^{\beta-\alpha}\right] d r \\
\leq \quad & k\|y\|_{\beta}\left\{\left\|f^{\prime}\right\|_{\infty}\|x-\tilde{x}\|_{s, t, \infty}(t-s)^{\beta}+\left\|f^{\prime}\right\|_{\infty}\|x-\tilde{x}\|_{s, t, \beta}(t-s)^{2 \beta}\right. \\
& \left.\quad+\left\|f^{\prime \prime}\right\|_{\infty}\|x-\tilde{x}\|_{s, t, \infty}\left[\|x\|_{s, t, \beta}+\|\tilde{x}\|_{s, t, \beta}\right](t-s)^{2 \beta}\right\} .
\end{aligned}
$$

Therefore

$$
\begin{aligned}
\|x-\tilde{x}\|_{s, t, \beta} \leq & k\|y\|_{\beta}\left\{\left\|f^{\prime}\right\|_{\infty}\|x-\tilde{x}\|_{s, t, \infty}+\left\|f^{\prime}\right\|_{\infty}\|x-\tilde{x}\|_{s, t, \beta}(t-s)^{\beta}\right. \\
& +\left\|f^{\prime \prime}\right\|_{\infty}\|x-\tilde{x}\|_{s, t, \infty}\left[\|x\|_{s, t, \beta}+\|\tilde{x}\|_{s, t, \beta}\right](t-s)^{\beta} \\
& \left.+\|f-\tilde{f}\|_{\infty}+\left\|f^{\prime}-\tilde{f}^{\prime}\right\|_{\infty}\|\tilde{x}\|_{s, t, \beta}(t-s)^{\beta}\right\} \\
& +k\|y-\tilde{y}\|_{\beta}\left[\|\tilde{f}\|_{\infty}+\left\|\tilde{f}^{\prime}\right\|_{\infty}\|\tilde{x}\|_{s, t, \beta}(t-s)^{\beta}\right] .
\end{aligned}
$$

Rearrange it to obtain

$$
\|x-\tilde{x}\|_{s, t, \beta} \leq k\left(1-k\left\|f^{\prime}\right\|_{\infty}\|y\|_{\beta}(t-s)^{\beta}\right)^{-1}\left\{\| y \| _ { \beta } \left[\left\|f^{\prime}\right\|_{\infty}\|x-\tilde{x}\|_{s, t, \infty}\right.\right.
$$




$$
\begin{aligned}
& +\left\|f^{\prime \prime}\right\|_{\infty}\|x-\tilde{x}\|_{s, t, \infty}\left[\|x\|_{s, t, \beta}+\|\tilde{x}\|_{s, t, \beta}\right](t-s)^{\beta} \\
+ & \left.\|f-\tilde{f}\|_{\infty}+\left\|f^{\prime}-\tilde{f}^{\prime}\right\|_{\infty}\|\tilde{x}\|_{s, t, \beta}(t-s)^{\beta}\right] \\
+ & \left.k\|y-\tilde{y}\|_{\beta}\left[\|\tilde{f}\|_{\infty}+\left\|\tilde{f^{\prime}}\right\|_{\infty}\|\tilde{x}\|_{s, t, \beta}(t-s)^{\beta}\right]\right\} .
\end{aligned}
$$

Set $\Delta=t-s$, and $A=k\left\|f^{\prime}\right\|_{\infty}\|y\|_{\beta}$. Then

$$
\begin{aligned}
\|x-\tilde{x}\|_{s, t, \infty} \leq & \left|x_{s}-\tilde{x}_{s}\right|+\|x-\tilde{x}\|_{s, t, \beta}(t-s)^{\beta} \\
\leq & \left|x_{s}-\tilde{x}_{s}\right|+k\left(1-A \Delta^{\beta}\right)^{-1} \Delta^{\beta}\left\{\| y \| _ { \beta } \left[\left\|f^{\prime}\right\|_{\infty}\|x-\tilde{x}\|_{s, t, \infty}\right.\right. \\
& +\left\|f^{\prime \prime}\right\|_{\infty}\|x-\tilde{x}\|_{s, t, \infty}\left[\|x\|_{s, t, \beta}+\|\tilde{x}\|_{s, t, \beta}\right] \Delta^{\beta} \\
& \left.+\|f-\tilde{f}\|_{\infty}+\left\|f^{\prime}-\tilde{f}^{\prime}\right\|_{\infty}\|\tilde{x}\|_{s, t, \beta} \Delta^{\beta}\right] \\
& \left.+k\|y-\tilde{y}\|_{\beta}\left[\|\tilde{f}\|_{\infty}+\left\|\tilde{f}^{\prime}\right\|_{\infty}\|\tilde{x}\|_{s, t, \beta} \Delta^{\beta}\right]\right\} .
\end{aligned}
$$

Denote

$$
B=k\|y\|_{\beta}\left(\left\|f^{\prime}\right\|_{\infty}+\left\|f^{\prime \prime}\right\|_{\infty}\left(\|x\|_{0, T, \beta}+\|\tilde{x}\|_{0, T, \beta}\right) T^{\beta}\right) .
$$

Then

$$
\begin{aligned}
\|x-\tilde{x}\|_{s, t, \infty} \leq & \left(1-\left(1-A \Delta^{\beta}\right)^{-1} \Delta^{\beta} B\right)^{-1} \\
& \times\left\{\left|x_{s}-\tilde{x}_{s}\right|+k\left(1-A \Delta^{\beta}\right)^{-1} \Delta^{\beta}\right. \\
& \times\left[\|y\|_{\beta}\left[\|f-\tilde{f}\|_{\infty}+\left\|f^{\prime}-\tilde{f}^{\prime}\right\|_{\infty}\|\tilde{x}\|_{s, t, \beta} \Delta^{\beta}\right]\right. \\
& \left.\left.+\|y-\tilde{y}\|_{\beta}\left[\|\tilde{f}\|_{\infty}+\left\|\tilde{f}^{\prime}\right\|_{\infty}\|\tilde{x}\|_{s, t, \beta} \Delta^{\beta}\right]\right]\right\} .
\end{aligned}
$$

Let $\Delta$ satisfy

$$
A \Delta^{\beta} \leq 1 / 3, \quad B \Delta^{\beta} \leq 1 / 3
$$

Namely, we take

$$
\Delta=\left(\frac{1}{3(A \vee B)}\right)^{1 / \beta}
$$

Then

$$
\|x-\tilde{x}\|_{s, t, \infty} \leq 2\left[\left|x_{s}-\tilde{x}_{s}\right|+C \Delta^{\beta}\right]
$$


where

$$
\begin{aligned}
C= & \frac{3}{2} k\left[\|y\|_{\beta}\left[\|f-\tilde{f}\|_{\infty}+\left\|f^{\prime}-\tilde{f}^{\prime}\right\|_{\infty}\|\tilde{x}\|_{s, t, \beta} \Delta^{\beta}\right]\right. \\
& \left.+\|y-\tilde{y}\|_{\beta}\left[\|\tilde{f}\|_{\infty}+\left\|\tilde{f}^{\prime}\right\|_{\infty}\|\tilde{x}\|_{s, t, \beta} \Delta^{\beta}\right]\right] .
\end{aligned}
$$

Applying the above estimate recursively we obtain

$$
\sup _{0 \leq r \leq T}\left|x_{r}-\tilde{x}_{r}\right| \leq 2^{n}\left[\left|x_{0}-\tilde{x}_{0}\right|+C \Delta^{\beta}\right],
$$

where $T=n \Delta$. Or we have

$$
\begin{aligned}
\sup _{0 \leq r \leq T}\left|x_{r}-\tilde{x}_{r}\right| \leq & 2^{k\left(\left\|f^{\prime}\right\|_{\infty} \vee\left(\left\|f^{\prime}\right\|_{\infty}+\left\|f^{\prime \prime}\right\|_{\infty}\left(\|x\|_{0, T, \beta}+\|\tilde{x}\|_{0, T, \beta}\right) T^{\beta}\right)\right)^{1 / \beta}\|y\|_{0, T, \beta}^{1 / \beta} T} \\
& \times\left\{\left|x_{0}-\tilde{x}_{0}\right|+\|y\|_{0, T, \beta}\left[\|f-\tilde{f}\|_{\infty}+\|x\|_{0, T, \beta}\left\|f^{\prime}-\tilde{f}\right\|_{\infty}\right]\right. \\
& \left.+\left[\|f\|_{\infty}+\|\tilde{f}\|_{\infty}\|x\|_{0, T, \infty}\right]\|y-\tilde{y}\|_{0, T, \beta}\right\} .
\end{aligned}
$$

\section{Stochastic differential equations driven by a fBm}

Let $B=\left\{B_{t}, t \geq 0\right\}$ be an $m$-dimensional fractional Brownian motion (fBm) with Hurst parameter $H>1 / 2$. That is, $B$ is a Gaussian centered process with the covariance function $E\left(B_{t}^{i} B_{s}^{j}\right)=R_{H}(t, s) \delta_{i j}$, where

$$
R_{H}(t, s)=\frac{1}{2}\left(t^{2 H}+s^{2 H}-|t-s|^{2 H}\right) .
$$

Consider the stochastic differential equation

$$
X_{t}=X_{0}+\int_{0}^{t} \sigma\left(X_{s}\right) d B_{s} .
$$

This equation has a unique solution (see [2] and 3]) provided $\sigma$ is continuously differentiable, and $\sigma^{\prime}$ is bounded and Hölder continuous of order $\lambda>\frac{1}{H}-1$. The stochastic integral is interpreted as a path-wise Riemann-Stieltjes integral.

Then, using the estimate (3.4) in Theorem 3.1 we obtain the following estimate for the solution of Equation (4.1), if we choose $\beta \in\left(\frac{1}{2}, H\right)$. Notice that $\frac{1}{\beta}<2$.

$$
\sup _{0 \leq t \leq T}\left|X_{t}\right| \leq 2^{k T\left(\left\|\sigma^{\prime}\right\|_{\infty} \vee|\sigma(0)|\right)\|B\|_{0, T, \beta}^{1 / \beta}\left(\left|X_{0}\right|+1\right) .}
$$


If $\sigma$ is bounded we can make use of the estimate (3.2) and we obtain

$$
\sup _{0 \leq t \leq T}\left|X_{t}\right| \leq\left|X_{0}\right|+k T\|\sigma\|_{\infty}\left\|\sigma^{\prime}\right\|_{\infty}^{\frac{1-\beta}{\beta}}\|B\|_{0, T, \beta}^{\frac{1}{\beta}} .
$$

These estimates improve those obtained by Nualart and Răşcanu in [3] based on a suitable version of Gronwall's lemma. The estimates (4.2) and (4.3) allow us to establish the following integrability properties for the solution of Equation (4.1).

Theorem 4.1 Consider the stochastic differential equation 4.1 . If $\sigma^{\prime}$ is bounded and Hölder continuous of order $\lambda>\frac{1}{H}-1$, then

$$
E\left(\sup _{0 \leq t \leq T}\left|X_{t}\right|^{p}\right)<\infty
$$

for all $p \geq 2$. If furthermore $\sigma$ is bounded, then

$$
E\left(\exp \lambda\left(\sup _{0 \leq t \leq T}\left|X_{t}\right|^{\gamma}\right)\right)<\infty
$$

for any $\lambda>0$ and $\gamma<2 \beta$.

If we apply these results to the linear equation satisfied by the derivative in the sense of Malliavin calculus of $X_{t}$ then we get that $X_{t}$ belongs to the Sobolev space $\mathbb{D}^{1, p}$ for all $p \geq 2$. This implies that if the coefficient $\sigma$ is infinitely differentiable with bounded derivatives of all orders, then, $X_{t}$ belongs to $\mathbb{D}^{\infty}$. This allows us to deduce the regularity of the density of the random vector $X_{t}$ at a fixed time $t>0$ assuming the following nondegeneracy condition:

(H) The vector space spanned by $\left\{\left(\sigma^{i j}\left(X_{0}\right)\right)_{1 \leq i \leq d}, 1 \leq j \leq m\right\}$ is $\mathbb{R}^{m}$.

That is, we have the following result.

Theorem 4.2 Consider the stochastic differential equation 4.1]). Suppose that $\sigma$ is infinitely differentiable with bounded derivatives of all orders, and the assumption (H) holds. Then, for any $t>0$ the probability law of $X_{t}$ has an $C^{\infty}$ density.

In 4. Nualart and Saussereau have proved that the random variable $X_{t}$ belongs locally to the space $\mathbb{D}^{\infty}$, and, as a consequence, they have derived the absolute continuity of the law of $X_{t}$ under the assumption $(\mathrm{H})$.

\section{References}

[1] Hu, Y. and Nualart, D. Rough path analysis via fractional calculus. Preprint 2006. 
[2] Lyons, T. Differential equations driven by rough signals (I): An extension of an inequality of L. C. Young. Mathematical Research Letters 1 (1994) 451-464.

[3] Nualart, D., Răşcanu, A. Differential equations driven by fractional Brownian motion. Collect. Math. 53 (2002) 55-81.

[4] Nualart, D., Saussereau, B. Malliavin calculus for stochastic differential equations driven by a fractional Brownian motion. Preprint.

[5] Samko S. G., Kilbas A. A. and Marichev O. I. Fractional Integrals and Derivatives. Theory and Applications. Gordon and Breach, 1993.

[6] Young, L. C. An inequality of the Hölder type connected with Stieltjes integration. Acta Math. 67 (1936) 251-282.

[7] Zähle, M. Integration with respect to fractal functions and stochastic calculus. I. Prob. Theory Relat. Fields 111 (1998) 333-374. 